\title{
Tratamiento de la amenaza de aborto con Anestesia Peridural continua*
}

\author{
Dr. Efraín González Montoya \\ Del Hospital de San José - Bogotá
}

El tratamiento de la amenaza de aborto con anestesia Peridural Continua, fue iniciado en el Hospital de San José de Bogotá, el 10 de Marzo de 1959.

Se originó al observar una paciente a quien se le aplicó una anestesia raquídea para practicarle un raspado uterino. La paciente fue mejor examinada y no se le encontró, como en un principio se creyó, un aborto en curso sino una amenaza de aborto.

No se le aplicó ningún otro tratamiento, distinto a la anestesia raquídea. La paciente mejoró totalmente de su amenaza de aborto.

Se comenzaron las experiencias con anestesia Peridural continua, con el ánimo de obtener efectos similares a los encontrados con la anestesia raquídea, sin los peligros de ésta.

\section{TECNICA DE LA ANESTESIA PERIDURAL}

La anestesia peridural comprende todos aquellos procedimientos en que se introducen medicamentos en el espacio peridural, por fuera del saco dural, con el propósito de bloquear los nervios raquídeos, bañando la solución anestésica las raíces o los troncos nerviosos en el punto en que sus cubiertas se hacen permeables.

El hecho de que estos métodos son dispendiosos desde el punto de vista técnico, explica probablemente la poca popularidad de que han gozado. Producen el bloqueo nervioso profundo rápidamente en los segmentos afectados. Existen varias vías de acceso

* Colaboró el Dr. Julián Buchelı, Jefe de Anestesia del Departamento de Obstetricia y Ginecología. 
al espacio peridural, para la aplicación de la anestesia. Su aplicación se lleva a cabo con una o más inyecciones en el espacio peridural, a través de los espacios interespinosos, en forma bastante similar a la anestesia raquídea.

La dificultad principal del bloqueo peridural surge de la necesidad de colocar la aguja directamente en el espacio peridural sin penetrar la duramadre. Como se sabe, el espacio se extiende solo 6 milímetros cuando más, desde el ligamento amarillo hasta la duramadre. Cuando se flexiona el raquis parece que se establece una presión negativa, fenómeno que se emplea como medio de reconocimiento de la punción peridural. Hay un método en que se emplea un pequeño manómetro de agua y otro que consiste en colocar la punta de la aguja en el sitio indicado y llevar a cabo la punción subaracnoidea y extraer entonces la aguja hasta el espacio peridural. Este método es objetable porque es posible que al realizar la inyección peridural se puedan introducir cantidades de solución anestésica intraduralmente.

Para llevar a cabo la punción peridural se selecciona usualmente el cuarto espacio lumbar y se esteriliza la piel. Se infiltra la piel por encima de la apófisis espinosa que limita por debajo el espacio, infiltrándose también el tejido subcutáneo. Se introduce la aguja de Touhy cuyo bisel es corto e incurvado, su calibre puede ser 16 o 18. Cuando la punta de la aguja se encuentra con los ligamentos interespinosos la penetración es difícil pero cuando penetra en el espacio peridural se nota una falta de resistencia. Una vez alcanzado el ligamento se extrae el mandril de la aguja y en el pabellón de ésta se coloca una gota de solución anestésica o solución salina. Se avanza poco la aguja en el interior de los ligamentos. Cuando la aguja ha penetrado en el espacio peridural se registra una presión negativa y la gota de agua es aspirada y desaparece del pabellón de la aguja.

Si el paciente está sentado se facilita la punción peridural, puesto que se obtiene la flexión adecuada de la columna vertebral.

También se usa otra prueba para saber si la punta de la aguja está en el espacio peridural: se inyecta con una jeringuilla aire y si la aguja no está en buen lugar, el aire no penetra, pero si está colocada adecuadamente, el émbolo no encuentra dificultad para hacer penetrar el aire. 
Una vez localizado el espacio peridural con los métodos antes indicados, se pasa a través de la aguja un catéter de polietileno logrando que penetre más o menos una pulgada dentro del espacio peridural, y si de antemano se ha dirigido la curvatura del bisel de la aguja hacia arriba lograremos que este catéter penetre en forma ascendente en el espacio peridural. A continuación se procede a retirar la aguja lentamente teniendo cuidado de que el catéter quede en su lugar. Se fija el catéter con esparadrapo y gasa.

La dosis inicial de anestésico debe administrarse con extremo cuidado, y se debe vigilar atentamente a la paciente puesto que puede presentar signos que indiquen la infiltración subaragnoidea de la solución anestésica. En la anestesia peridural el bloqueo motor de las extremidades inferiores se presenta más tarde y puede que no sea completo a menos que se trate especialmente de bloquear el plejo lumbar. Este fenómeno constituye el fundamento de una prueba para comprobar la localización de la punta del catéter. En todas las técnicas se administra una pequeña dosis de prueba de la solución anestésica antes de inyectar la dosis usual. Después de un lapso de 5 minutos se examina la paciente para buscar signos de parálisis motora del miembro inferior; si la dosis de prueba produce parálisis motora se ha llevado a cabo la punción subaragnoidea. Si por el contrario, se conserva el movimiento, se puede estar seguro que la inyección ha sido peridural.

Después que el catéter ha sido colocado y fijado en su lugar se administra una dosis de prueba de 5 c.c. de xilocaina al $2 \%$ sin epinefrina. Se dejan transcurrir 5 minutos y a la terminación de este período se le pregunta a la paciente si puede mover los dedos de los pies. Cuando la dosis de prueba ha sido inyectada periduralmente, la paciente no es capaz de notar efecto alguno en ningún lado; el movimiento de los pies comprueba que los 5 c.c. no fueron administrados en el espacio subaragnoideo. Como precaución adicional se puede investigar la presencia de anestesia en el área sacra: el resultado debe ser negativo.

Si la dosis de prueba no produce efectos, se puede administrar lentamente la dosis anestesiante inicial.

Se han empleado diferentes anestésicos en el bloqueo peridural. El anestésico ideal para esta clase de anestesia debe poseer las siguientes características: el bloqueo sensitivo debe ser rápi- 
do y completo; el período de efecto debe ser uniforme y la duración bastante prolongada; no deben presentarse sensaciones desagradables durante los períodos de anestesia ni en la recuperación. La eliminación debe ser rápida; no debe presentar efectos tóxicos y el restablecimiento de la función nerviosa debe ser completo.

Para el tratamiento de la amenaza de aborto y del parto prematuro hemos usado la xilocaína en solución al $2 \%$ sin epinefrina. Inicialmente pensamos usar la xilocaína en jalea dentro del espacio peridural pero inconvenientes de esterilización nos hicieron desechar este sistema. Las dosis que usamos fueron: como prueba 5 c.c. de la solución de xilocaína al $2 \%$ y como dosis de mantenimiento 6 c.c. de xilocaína al $2 \%$ sin epinefrina, cada 4 horas.

Solo en dos casos rebeldes tuvimos que usar goteo continuo preparando una solución al $0.5 \%$ en 500 c.c. de solución salina fisiológica.

\section{CASO No 1}

Mayo 13 de 1959 - R. L. P. de E. - Edad 40 años. - Embarazo No 13

Consulta por dolor lumbar suprapúblico y hemorragia vaginal.

Antecedentes obstétricos: 12 embarazos, 10 a término con partos normales. Dos abortos por causa desconocida.

Antecedentes ginecológicos: Menarquia a los 11 años. Ciclo 30 x 4 . En amenorrea desde su último parto, hace un año. Tensión Arterial 120 x 80 . Pulso $88 / \mathrm{m}$. Al examen general no se encuentra nada anormal.

Examen Obstétrico: Utero aumentado de tamaño $11 \mathrm{~cm}$. doloroso a la palpación. Tacto vaginal: genitales externos normales; en la vagina se encuentra gran cantidad de coágulos, cuello uterino central, blando, largo y cerrado.

Examen de Laboratorio: Galli Mainini Positivo.

Edad del embarazo, 3 meses.

Diagnóstico: Amenaza de aborto.

Tratamiento: El día 13 de Mayo a las 10:30. Se aplica anestesia peridural continua. Dosis total 27 c.c. de Xilocaína al $2 \%$ durante 28 horas. Las contracciones uterinas dolorosas desaparecieron a los 15 minutos de iniciado el tratamiento, y a las 12 horas la hemorragia vaginal desapareció totalmente.

La paciente sale del hospital a los 4 días, en buenas condiciones. Su control prenatal demuestra normalidad.

\section{CASO No 2}

Junio 10 de 1959. - M. A. T. de V. - Edad 37 años. - Embarazo No 4. 
Consulta por hemorragia vaginal abundante y dolores abdominales tipo cólico.

Antecedentes Obstétricos: 3 embarazos con partos normales a término. El último hace 14 años.

Antecedentes Ginecológicos: Menarquia a los 13 años. Ciclo 24 x 4. Flujo vaginal abundante de color blanco. Ultima menstruación 15 de Diciembre de 1958.

Temperatura 38,7 gr. Pulso 80/m. Tensión Arterial $100 \times 60$.

Examen Obstétrico: Altura uterina $20 \mathrm{cms}$. Tacto vaginal: vagina amplia, cuello uterino blando central con $1 \mathrm{~cm}$. de dilatación, sale sangre en cantidad apreciable.

Edad del embarazo, 51/2 meses.

Tratamiento: El día 10 de Junio de 1959 a las 11:30 se comienza la anestesia peridural continua. Se aplican 12 c.c. de Xilocaína al $2 \%$ en 12 horas. La hemorragia cede a las 6 horas de tratamiento. La paciente presenta dolor en el punto de inserción del catéter.

La paciente sale del hospital a los 3 días en buenas condiciones. Su estado prenatal permanece normal.

\section{CASO No 3}

Junio 11 de 1959. - U. V. de V. - Edad 34 años. - Embarazo No 8.

La paciente consulta por hemorragia vaginal y contracciones uterinas de tipo cólico menstrual.

Antecedentes Obstétricos: Siete embarazos. Cinco a término normales y dos abortos.

Antecedentes Ginecológicos: Menarquia a los 15 años. Ciclo 30 x 4. Ultima menstruación el 2 de Marzo.

Temperatura 37,2 grados. Pulso 100/m. Tensión Arterial 150 x 90. Se encuentra la paciente deshidratada y en estado general bastante regular.

Examen Obstétrico: Utero aumentado de tamaño $(4 \mathrm{~cm}$.) doloroso. Tacto Vaginal: Genitales Externos con abundante secreción hemorrágica. Vagina llena de coágulos. Cuello central grueso con $3 \mathrm{~cm}$. de dilatación.

Edad del embarazo, 3 meses.

Diagnóstico: Aborto en curso.

Tratamiento: El día 11 de Junio de 1959 a las 14 horas se aplica anestesia peridural continua, dosis total 10 c.c. durante 7 horas. Al finalizar las 7 horas de tratamiento la paciente presenta su aborto y se practica raspado uterino.

\section{CASO No 4}

Junio 13 de 1959. - O. V. de R. - Edad 27 años. - Embarazo No 8. 
Consulta por hemorragia vaginal en regular cantidad, dolor abdominal tipo cólico.

Antecedentes Obstétricos: 4 embarazos a término con partos normales, 3 abortos en los últimos 3 embarazos.

Antecedentes Ginecológicos: Menarquia a los 14 años. Ciclo muy irregular. Ultima menstruación 19 de Marzo de 1959.

Temperatura 36,2 grados. Pulso $90 / \mathrm{m}$. Tensión Arterial 130 x 80 . Clínicamente no se encuentra nada más anormal.

Examen Obstétrico: Utero aumentado de tamaño $(11 \mathrm{~cm}$.) de consistencia y caracteres normales. Tacto vaginal. Genitales externos normales. Cuello blando central largo y cerrado. Se aprecia hemorragia que sale por el orificio cervical, en regular cantidad.

Edad de embarazo $2 \frac{1}{2}$ meses.

Diagnóstico: Amenaza de Aborto.

Tratamiento: El día 13 de Junio a las 14:45 se comienza la anestesia peridural continua. Dosis total 13 c.c. de Xilocaína al $2 \%$. Horas de tratamiento, 26.

Las contracciones uterinas dolorosas desaparecieron a los 30 minutos de aplicada la anestesia. La hemorragia no continuó después de las 24 horas. El embarazo continúa en perfectas condiciones.

\section{CASO $\mathbf{N}^{0} 5$}

Junio 21 de 1959. - B. F. de M. - Edad 27 años. - Embarazo No 5.

La paciente consulta por hemorragia vaginal oscura y de olor fétido.

Antecedentes Obstétricos: 6 embarazos a término con partos normales.

Antecedentes Ginecológicos: Menarquia a los 15 años, ciclo 30 x 4 . Ultima menstruación, 26 de Enero de 1959.

Antecedentes personales: Litiasis biliar, que fue comprobada por radiografías de vías biliares, durante este embarazo.

Temperatura 36,3 grados. Pulso $86 / \mathrm{m}$. Tensión Arterial 140 x 80 . Intolerancia por las grasas. Dolor en el punto vesicular.

- Examen Obstétrico: Utero grávido. Altura $14 \mathrm{~cm}$. de consistencia normal. Tacto vaginal: Cuello largo, blando y cerrado. Presenta hemorragia oscura.

Diagnóstico: Amenaza de aborto.

Tratamiento: Se aplica anestesia peridural continua el día 24 de Junio a las 15:30 horas. Durante 36 horas. Dosis total 32 c.c. de Xilocaína. La paciente abortó al finalizar las 36 horas de tratamiento un feto muerto y macerado.

La anestesia produjo disminución de la hemorragia uterina.

\section{CASO $\mathbf{N}^{0} 6$}

Junio 21 de 1959. - B. F. de M. - Edad 27 años. - Embarazo No 5.

Consulta por hemorragia vaginal en regular cantidad desde hace 20 días, sin dolor. 
Antecedentes Obstétricos: 3 partos a término, normales. Un aborto de dos meses, en el último embarazo

Antecedentes Ginecológicos: Menarquia a los 15 años. Ciclo 30 x 3. Temperatura 37 grados. Pulso $90 / \mathrm{m}$. Tensión arterial $120 \times 80$. Se encuentra soplo sistólico grado II en foco aórtico.

Examen Obstétrico: Utero de $12 \mathrm{~cm}$. doloroso a la palpación. Tacto vaginal: Cuello largo con desgarros laterales, cerrado; la sangre sale por el orificio cervical.

Edad del embarazo, 4 meses.

Tratamientos anteriores: Se aplicaron dosis altas de Beta Glicerofosfato de sodio, sin ningún resultado.

Tratamiento: Anestesia peridural continua, el día 21 de Junio desde las 0:30. Total de horas de tratamiento, 72. Dosis total de anestésico empleado 38 c.c. de Xilocaína al $2 \%$. La hemorragia desapareció a las 17 horas de tratamiento.

El tratamiento se continuó casi en forma ambulatoria, pues la paciente no estaba acostada.

Su control prenatal demuestra buen estado cbstétrico.

\section{CASO No 7}

H. C. V. de G. - Edad 36 años. - Embarazo No 2.

Consulta por hemorragia vaginal y dolores tipo cólico.

Antecedentes Obstétricos: Un embarazo con aborto a los $3 \frac{1}{2}$ meses.

Antecedentes Ginecológicos: Menarquia a los 15 años. Ciclo 28 x 4 . Dismenorrea.

Antecedentes Quirúrgices: Apendicectomía.

Ultima menstruación 5 de Enero de 1959. Pulso $80 / \mathrm{m}$. Tensión Arterial $120 \times 80$. No se encuentra nada anormal. Signos de embarazo.

Examen Obstétrico: Utero aumentado de tamaño. Altura $16 \mathrm{~cm}$. Ruidos cardíacos fetales normales. Tacto vaginal: genitales externos normales. Presenta hemorragia vaginal. Cuello central blando sin dilatación.

Tratamiento: Se aplica anestesia peridural continua durante 14 horas. Dosis total de Xilocaína al 2\% 13 c.c. El dolor y la hemorragia cedieron al tratamiento a las 6 horas. Presentó dolor en el punto donde estaba el catéter.

El embarazo continúa normalmente.

\section{CAsO № 8}

N 9108. - Junio 18 de 1959. - A. P. de C. - Edad 29 años. Embarazo No 3

Consulta por hemorragia vaginal, acompañada de dolor tipo cólico, en la región inguinal y supra-púbica.

Antecedentes Obstétricos: Dos embarazos a término normales.

Antecedentes Ginecológicos: Menarquia a los 14 años. Ciclo 25 x 3. Ultima menstruación 23 de Mayo de 1959. Temperatura 36 grados. Pulso 68/m. 
Tensión Arterial 120 x 80. Presenta arritmia cardíaca.

Lxamen Obstétrico: Genitales Externos normales. Tacto vaginal: cuello largo y cerrado, sale hemorragia por el orificio cervical. Utero aumentado de tamaño.

Edad de embarazo, 45 días.

Examen de laboratorio: Galli Mainini Positivo.

Diagnóstico: Amenaza de aborto.

Tratamiento: Anestesia peridural continua durante $25 \frac{1}{2}$ horas. Dosis total: 24 c.c. de Xilocaína al $2 \%$.

La paciente deja de sangrar a las 12 horas de tratamiento.

En el control prenatal el embarazo continúa normal.

\section{CASO $\mathbf{N}^{0} 9$}

N 9416. - G. Z. - Edad 25 años. - Embarazo No 2.

La paciente consulta por hemorragia vaginal desde el día de ayer.

Antecedentes Obstétricos: Un parto espontáneo normal.

Antecedentes Ginecológicos: Menarquia a los 16 años. Ciclo 30 x 3. Ultima menstruación Marzo $1^{\text {o }}$ de 1959. Pulso 65/m. Tensión Arterial 140 x 80. Temperatura 36,2 grados. Clínicamente no se encuentra nada anormal.

Examen Obstétrico: Utero aumentado de tamaño $(14 \mathrm{~cm}$.) Tacto vaginal: cuello blando, largo, central, cerrado; presenta secreción hemorrágica por el orificio cervical uterino.

Edad del embarazo, 3 meses 20 días.

Tratamiento: El día $1^{0}$ de Julio de 1959 le fue aplicada la anestesia peridural continua durante 14 horas, con niveles de cmbligo a periné con una dosis total de 12 c.c. de Xilocaína al $2 \%$.

La hemorragia desapareció totalmente a las 8 horas. La paciente sale del hospital en buenas condiciones.

El embarazo continúa en perfecta evolución.

\section{CASO No 10}

$$
\mathrm{N}-1459 \text { - A. M. de C. - Edad } 22 \text { años. - Embarazo No } 1 .
$$

La paciente consulta por hemorragia vaginal intermitente, acompañada de dolores hipogástricos.

Antecedentes Ginecológicos: Menarquia a los 15 años ciclo 30 x 5 . Dismenorrea. Flujo vaginal de color amarillo. Ultima regla 10 de Enero de 1959. Temperatura 37 grados. Pulso 90/m. Tensión Arterial 130 x 90. Aparatos Respiratorio, Circulatorio, Digestivo, Urinario: Clínicamente normales.

Examen Obstétrico: Genitales externos normales. Se encuentra sangre en la vagina. Tacto vaginal: Vagina estrecha, cuello uterino central en forma de embudo, se aprecia salida de sangre por el orificio cervical. Utero aumentado de tamaño. 
Examen de Laboratorio: Galli Mainizi Positivo,

Diagnóstico: Amenaza de aborto.

Tratamiento: Se aplica anestesia peridural continua durante 26 horas con dosis total de Xilocaína al $2 \%, 20$ c.c. La hemorragia cesa a las 8 horas. La evolución del embarazo continúa normalmente.

\section{CASO $\mathbf{N}^{0} 11$}

N - 9626. - M. T. H. de G. - Edad 37 años. - Embarazo No 10.

La paciente consulta por hemorragia vaginal y dolores en la región sacra.

Antecedentes Obstítricos: 9 embarazos, 6 embarazos con partos a término normales. 3 abortos.

Antecedentes Ginecológicos: Menarquia a los 13 años. Ciclo 30 x 3. Flujo vaginal amarillo. Ultima menstruación el 14 de Abril de 1959.

Pulso 88/m. Tensión Arterial 140 x 90. Temperatura 36,7 grados. Aparatos Digestivo, Circulatorio, Respiratorio, Clínicamente normales.

Examen Obstétrico: Genitales Externos normales. Tacto vaginal: Cuello de multípara, orjficio interno cerrado. Presenta desgarros laterales. Utero aumentado de tamaño $(11 \mathrm{~cm}$.) correspondiendo a 21/2 meses de embarazo.

Tratamiento: El día 9 de Julio se hospitaliza para la aplicación de anestesia peridural continua. Dosis total: 30 c.c. de Xilocaína al 2\%. Durante 42 horas. La sintomatología de la amenaza de aborto desaparece totalmente.

El embarazzo continúa normalmente.

\section{CASO $\mathrm{N}^{0} 12$}

Julio 11 de 1959. - N 9669. - Edad 33 años. - Embarazo № 12. L. P. le C. Consulta por hemorragia vaginal y dolores hipogástricos.

Antecedentes Obstétricos: 10 embarazos con partos a término normales. 1 aborto de tres meses.

Antecedentes Ginecológicos: Menarquia a los 14 años. Ciclo menstrual 30 x 3 Hipomenorrea. Flujo vaginal blanco. Ultima menstruación 18 de Febrero de 1959 .

Examen Físico General: Temperatura 37 grados. Pulso 78/m. Tensión Arterial $100 \times 60$. Aparatos Digestivo, Urinario, Circulatorio, Respiratorio, normales.

Examen Obstétrico: Genitales externos normales. Vagina estrecha de paredes normales; se encuentra sangre en cantidad apreciable. Cuello del útero hipertrófico con desgarros laterales, largo, cerrado. Utero aumentado de tamaño $14 \mathrm{~cm}$.

Edad del embarazo, 41/2 meses.

Diagnóstico: Amenaza de aborto.

Tratamiento: El día 11 de Julio de 1959 se aplica anestesia peridural continua. Dosis total 36 c.c. de Xilocaína al $2 \%$ durante 46 horas. Los dolores ab- 
dominales desaparecieron a los 15 minutos, y la hemorragia cesa a las 16 horas.

La paciente sale del hospital en buenas condiciones y la evolución del embarazo es normal.

\section{CASO No 13}

Julio 14 de 1959. - A. G. de G. — N 9754. - Edad 29 años. — Embarazo No 9.

La paciente consulta por hemorragia vaginal que le apareció hace dos días. Sin dolor.

Antecedentes Obstétricos: La paciente ha tenido 8 embarazos de los cuales, los 4 primeros fueron normales con partos a término, los cuatro últimos han terminado en abortos.

Antecedentes Ginecológicos: Menarquia a los 14 años. Ciclo 30 x 3 . Flujo vaginal de color amarillo. Ultima menstruación 27 de Marzo de 1959. Temperatura 37 grados. Pulso $78 / \mathrm{m}$. Tensión Arterial $135 \times 85$. Aparato Digestivo, Respiratorio, Urinario, Cardiovascular, clínicamente normales.

Examen Obstétrico: Genitales externos normales. Tacto vaginal: Vagina normal. Cuello largo, blando, cerrado sale sangre por el orificio cervical. Utero aumentado de tamaño $14 \mathrm{~cm}$.

Diagnóstico: Amenaza de aborto.

Tratamiento: El día 14 de Julio de 1959 se le aplica anestesia peridural continua, con Xilocaína al $2 \%$ durante 36 horas; dosis total 31 c.c. La hemorragia que drenaba por el orificio cervical cesó a las 20 horas de tratamiento.

El embarazo continúa normalmente.

\section{CASO No 14}

Julio 14 de 1959. - H. C. de C. - Edad 32 años. - N $9778-$ Embarazo No 7.

La paciente consulta por hemorragia vaginal muy abundante, y dolores hipogástricos, tipo cólico.

Antecedentes Obstétricos: 6 embarazos normales con partos eutósicos.

Antecedentes Ginecológicos: Menarquia a los 15 años. Ciclo 30 x 3. Ultima menstruación 15 de Mayo de 1959. Temperatura 35,8 grados. Tensión Arterial $90 \times 60$. Pulso $100 / \mathrm{m}$. Conjuntivas anémicas. Pulso delgado y filiforme. Ruidos cardíacos velados.

Examen Obstétrico: Genitales externos normales. Tacto vaginal, cuello central, largo, cerrado. Matriz aumentada de tamaño, lisa, blanda, regular. Se aprecia abundante hemorragia.

Examen de Laboratorio: Hematíes 2'350.000. Leucocitos 4.300. Hemoglobina $31 \%$. Galli Mainini Positivo.

Edad del embarazo, 2 meses.

Diagnóstico: Amenaza de Aborto. 
Tratamiento: Se aplica anestesia peridural continua. Durante 72 horas. Dosis total 24 c.c. de Xilocaína al $2 \%$. Sangre total 500 c.c.

La hemorragia desapareció totalmente a las 50 horas de tratamiento. El embarazo continúa normalmente.

\section{CASO No 15}

Julio 31 de 1959. - R. N. de T. - N 1022 - Edad 32 años. Embarazo No 5

La paciente consulta por hemorragia vaginal discreta, con dolor tipo cólico.

Antecedentes Obstétricos: 4 embarazos con partos a término normales.

Antecedentes Ginecológicos: Menarquia a los 15 años. Ciclo 30 x 5. Dismenorrea. Flujo vaginal abundante de color amarillo. Ultima menstruación el 1 o de Julio de 1959.

Temperatura 36,7 grados. Pulso 90/m. Tensión Arterial $110 \times 80$. Aparato Digestivo, Cardiovascular, Respiratorio, Urinario, clínicamente normales.

Examen Obstétrico: Genitales externos normales. Tacto vaginal, cuello central, largo, cerrado. Utero aumentado de tamaño correspondiendo a un embarazo de 50 días.

Examen de Laboratorio: Galli Mainini Positivo.

Edad del embarazo 50 días.

Diagnóstico: Amenaza de aborto.

Tratamiento: Se aplica anestesia peridural continua. Dosis total 23 c.c. de Xilocaína al $2 \%$. Durante un período de 15 horas.

Los síntomas de la amenaza de aborto se dominaron rápidamente y el embarazo cơntinúa en buenas condiciones.

\section{CASO No 16}

Agosto 5 de 1959. - C. G. de F. - N 10406 - Edad 33 años. - Embarazo No 6 .

La enferma consulta porque en el día de hoy presentó una fuerte hemorragia vaginal con expulsión de coágulos, con dolores tipo cólico.

Antecedentes Quirúrgicos: Apendicectomía. Amigdalectomía.

Antecedentes Ginecológicos: Menarquia a los 14 años ciclo 30 x 4 .

Antecedentes Obstétricos: 5 embarazos. 2 a término normales de los cuales el último fue gemelar. Desde el parto gemelar ha presentado 3 abortos. Ultima menstruación el 2 de Junio de 1959.

Temperatura 36,1 grados. Pulso $86 / \mathrm{m}$. Tensión arterial $115 \times 75$. Aparato Respiratorio, Circulatorio, Digestivo, clínicamente normales.

Examen Obstétrico: Genitales externos normales. Tacto vaginal: Vagina de paredes normales. Cuello hipertrófico con grandes desgarros laterales, sale sangre por el orificio cervical. Utero aumentado de tamaño, que corresponde a 2 meses de embarazo. 
Tratamiento: Se aplica anestesia peridural continua. Dosis total: 78 c.c. Xilocaína al $2 \%$. Durante 96 horas.

La hemorragia vaginal no le calmó en ningún momento durante el tratamiento con anestesia peridural. A las 96 horas de tratamiento llegó el resultado de un Galli Mainini, que resultó Negativo.

Más tarde se practicó un raspado uterino.

\section{CASO No 17}

Agosto 8 de 1959. - A. T. B. de R. - N 10451 - Edad 39 años - Embarazo $\mathrm{N}^{\circ} 8$.

La paciente consulta por hemorragia vaginal cue le comenzó el día 7 de Agosto con dolores hipogástricos fuertes.

Antecedentes Obstétricos: 6 embarazos con fetos vivos y a término 1 aborto de 5 meses.

Antecedentes Ginecológicos: Menarquia a los 14 años ciclo 20 x 4 .

Antecedentes Médicos: Sarampión, viruela. Temperatura 37 grados. Pulso $90 / \mathrm{m}$. Tensión Arterial $100 \times 60$. Aparatos Digestivo, Respiratorio, Cardiovascular, Urinario, clínicamente normales.

Examen Obstétrico: Genitales externos normales. Tacto vaginal, cuello central, blando y cerrado. Vagina normal. Utero con una altura de $14 \mathrm{~cm}$.

Diagnóstico: Amenaza de aborto.

Tratamiento: Se aplica anestesia peridural continua durante 36 horas. Dosis total 30 c.c. de Xilocaína al $2 \%$.

La hemorragia y los dolores se calmaron totalmente.

En el control prenatal el embarazo continúa normalmente.

\section{CASO $\mathbf{N}^{0} 18$}

Agosto 9 de 1959. - B. de R. - N 10482

La paciente ingresa al Servicio por hemorragia vaginal.

Antecedentes Obstétricos: 5 embarazos con fetos vivos y a término, partos normales.

Antecedentes Ginecológicos: Menarquia a los 14 años ciclo 30 x 6 . Hipermenorrea. Flujo vaginal de color blanco. No sabe cuándo fue su última regla. Temperatura 36,8 grados. Pulso 76/m. Tensión Arterial 100 x 80.

En el examen de los aparatos Respiratorio, Circulatorio, Digestivo, Urinario, no se encontró nada anormal.

Examen Obstétrico: Genitales externos. Desgarro perineal de II grado. Tacto vaginal: Cuello largo cerrado, un poco posterior. Utero con una altura de $8 \mathrm{~cm}$.

Diagnóstico: Amenaza de aborto.

Tratamiento: Anestesia peridural continua. Dosis total 12 c.c. de Xilocaína al $2 \%$. Horas de tratamiento 24 . 
La hemorragia desapareció totalmente. El control prenatal confirmó el embarazo, el cual continúa normalmente.

\section{CASO No 19}

Agosto 24 de 1959 - J. M. de C. - N 6462 - Edad 23 años. - Embara$70 \mathrm{~N} \div 3$.

Consulta por hemorragia vaginal y dolor tipo cólico.

Antecedentes Obstétricos: 2 embarazos normales con partos a término normales.

Antecedentes Ginecológicos: Menarquia a los 18 años ciclo 30 x 4 . Ultima menstruación el 29 de Junio de 1959. Paciente en buenas condiciones generales; pulso $80 / \mathrm{m}$. Tensión arterial $100 \times 70$.

Examen Obstétrico: Genitales externos normales. Tacto vaginal: Vagina estrecha, cuello uterino largo cerrado, blando. Utero aumentado de tamaño que corresponde a dos meses de embarazo.

Tratamiento: Se aplica anestesia peridural continua durante 24 horas. Dosis total de Xilocaína al $2 \% 18$ c.c. A las 24 horas de tratamiento la paciente deja de sangrar y ha desaparecido el dolor hipogástrico. Sale del Hospital el día 27 de Agosto en buenas condiciones.

\section{COMENTARIO}

En el presente trabajo, las pacientes no fueron seleccionadas, practicándose la anestesia peridural sin discriminación a aquellas que presentaban los signos y síntomas de la amenaza de aborto.

La edad varía de los 17 años a los 40 . La mayoría de las mujeres se encuentran en la tercera década de la vida.

El síntoma que predomina en la consulta es la hemorragia vaginal. Le sigue en frecuencia el dolor tipo cólico de localización hipogástrica.

En el examen físico general de las pacientes no se encontró clínicamente la causa que pudiera estar ocasionando la amenaza de aborto.

En el examen obstétrico de una paciente se encontró un cuello uterino incontinente. En las demás no se halló nada anormal.

El aparato Respiratorio y el Sistema Cardiovascular durante la anestesia peridural continua, no se modificaron clínicamente. 
El único inconveniente que se notó en algunos casos fue el dolor que se presentó en el punto de inserción del catéter de polietileno.

En la mayoría de los casos el dolor de tipo cólico desapareció rápidamente después de haberse aplicado anestesia peridural. La hemorragia uterina desaparece casi siempre a las pocas horas de aplicada la anestesia.

Se puede presentar esta hipótesis para explicar el efecto beneficioso de la anestesia peridural en la amenaza de aborto: el aborto puede ocurrir al tener el útero una incoordinación de la contractilidad, presentándose así una fibrilación muscular. La anestesia peridural transformaría, la fibrilación uterina, en una contractilidad normal, también disminuiría el tono uterino, aumentaría la oxigenación, produciendo sedación de la paciente.

Otro punto de acción de la anestesia peridural sería bloqueando la inervación simpática del útero directamente por el nervio presacro, e indirectamente a través de las cápsulas suprarrenales, que descargan adrenalina. Se ha visto que la adrenalina desorganiza e inhibe una actividad uterina previamente normal. La anestesia peridural, al bloquear la inervación simpática del útero y de las suprarrenales, pondría fin a su acción y normalizaría la contracción uterina. 